\title{
Virginia IIIIT)ch Research on Persistent Pain in Late Life: Current Topics and Challenges
}

\section{Alissa Kate Holland, Karen A. Roberto, \& Susan N. Perkins}

\section{INTRODUCTION}

Background: Living with chronic diseases and their manifestations affects daily functioning and influences the quality of life of many older individuals. One frequently associated symptom and consequence of these diseases is persistent pain.

Purpose: Building upon comprehensive review of the multidisciplinary pain and aging literature published between 1990 and 1998, (Roberto \& Gold, 2001), the aim of this research is to provide an update and overview of the geriatric pain literature published from 1999 through 2004

Research questions:

1. What type of samples and procedures are used in the study of persistent pain in late life?

2. What is the focus of the current empirical research on persistent pain in later life?

3. What are the primary methods prescribed to treat and manage persistent pain in late life?

4. How much attention is given to functional and psychological issues that influence the quality of life of older adults living with persistent pain?

\section{METHOD}

Article Search: Medline and PsychLit

Keywords: (a) elderly, (b) aging, (c) pain

Restrictions: Focus on humans; Published in English

Database: 185 empirical or data-driven articles analyzed

Complete reference list available at:

www.gerontology.vt.edu/research/center_research.htm

Coding scheme: 14 major categories:

- type of article

- type of data

- study design

- sample

- psych manifestations

- social

issues/outcomes

- coping

- functional outcomes

- location of pain

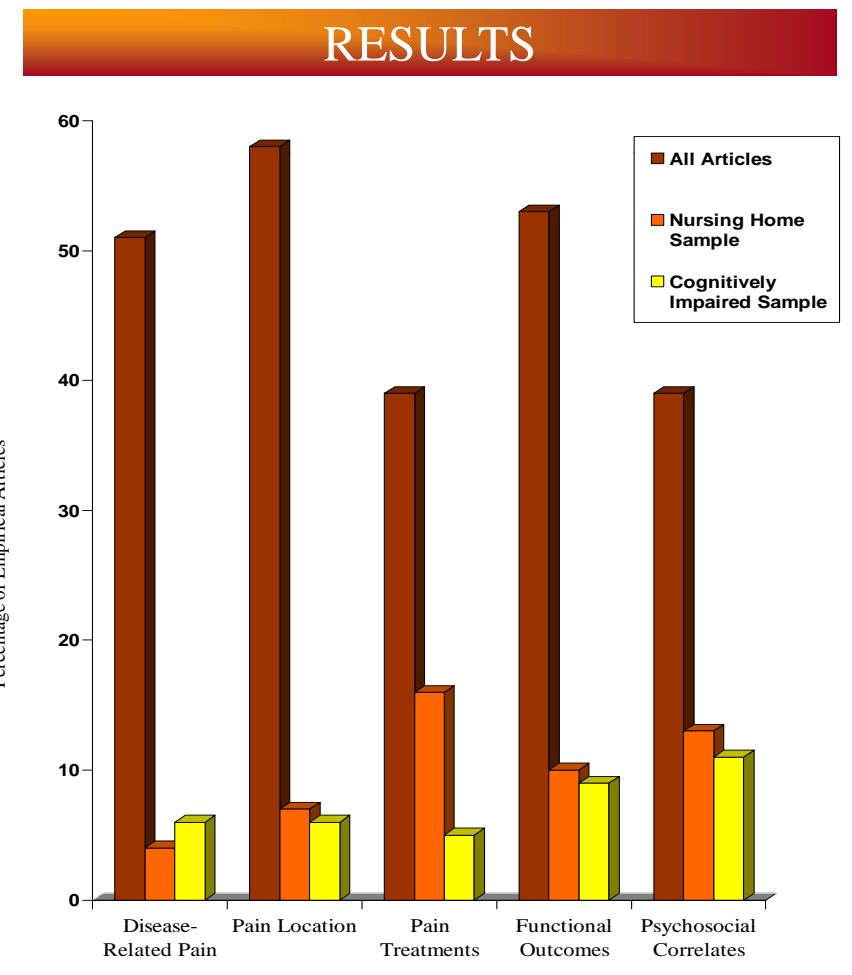

Table 1: Pain Topics
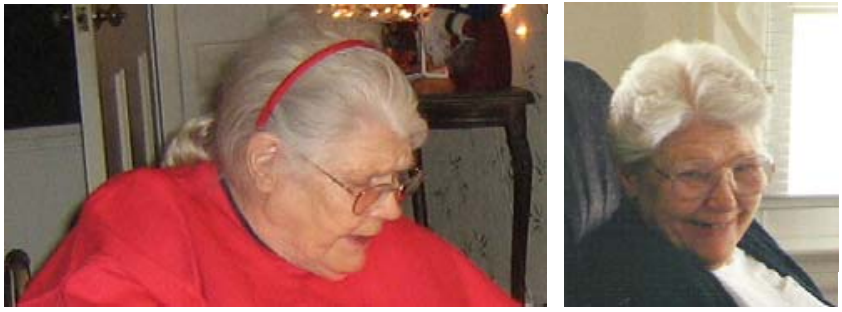

Figure 1: Persistent pain as experienced by an older female adult

\section{RESULTS}

- The majority of samples were comprised primarily of cognitively intact older adults living in the community (86\%).

- Most researchers (89\%) included both older women and men in their samples; however, in these studies it was common for women to significantly outnumber men.

- Cross-sectional (51\%; n=94) and experimental designs (29\%; $n=53$ ) were most often used to investigate the various aspects of persistent pain in late life.

- Fifty-one percent ( $n=94)$ of the articles discussed pain as a cause or consequence of a specific disease. Authors most often addressed a single disease entity $(n=71 ; 76 \%)$.

- Fifty-eight percent $(\mathrm{n}=107)$ of the articles explicitly discussed persistent pain in certain areas of the body. Hip and knee pain received the greatest amount of attention $(n=43$; $23 \%$ of all empirical articles)

- Seventy-three empirical articles (39\%) described treatments for persistent pain in late life. Pharmacological treatments ( $\mathrm{n}=$ 32; 44\%) were the most widely discussed.

- Functional limitations associated with persistent pain were discussed in 98 (53\%) of the articles.

-Thirty-nine percent $(\mathrm{n}=72)$ of the articles explicitly address psychological issues related to persistent pain.

\section{Conclusions}

- As the complex issues surrounding persistent pain in late life are being untangled:

- New areas of emphasis have emerged such as the recognition of

body mass and weight; factors especially prevalent in old women.

- Other areas have expanded including the focus on pain among elders with cognitive impairments and the use of alternatives and additions to pharmacologic treatments.

- Research is needed to develop evidence-based treatments, and address the psychosocial correlates and consequences of pain.

- Researchers and practitioners are in prime positions to advance the study of persistent pain in late life and ultimately, improve pain care practices. 


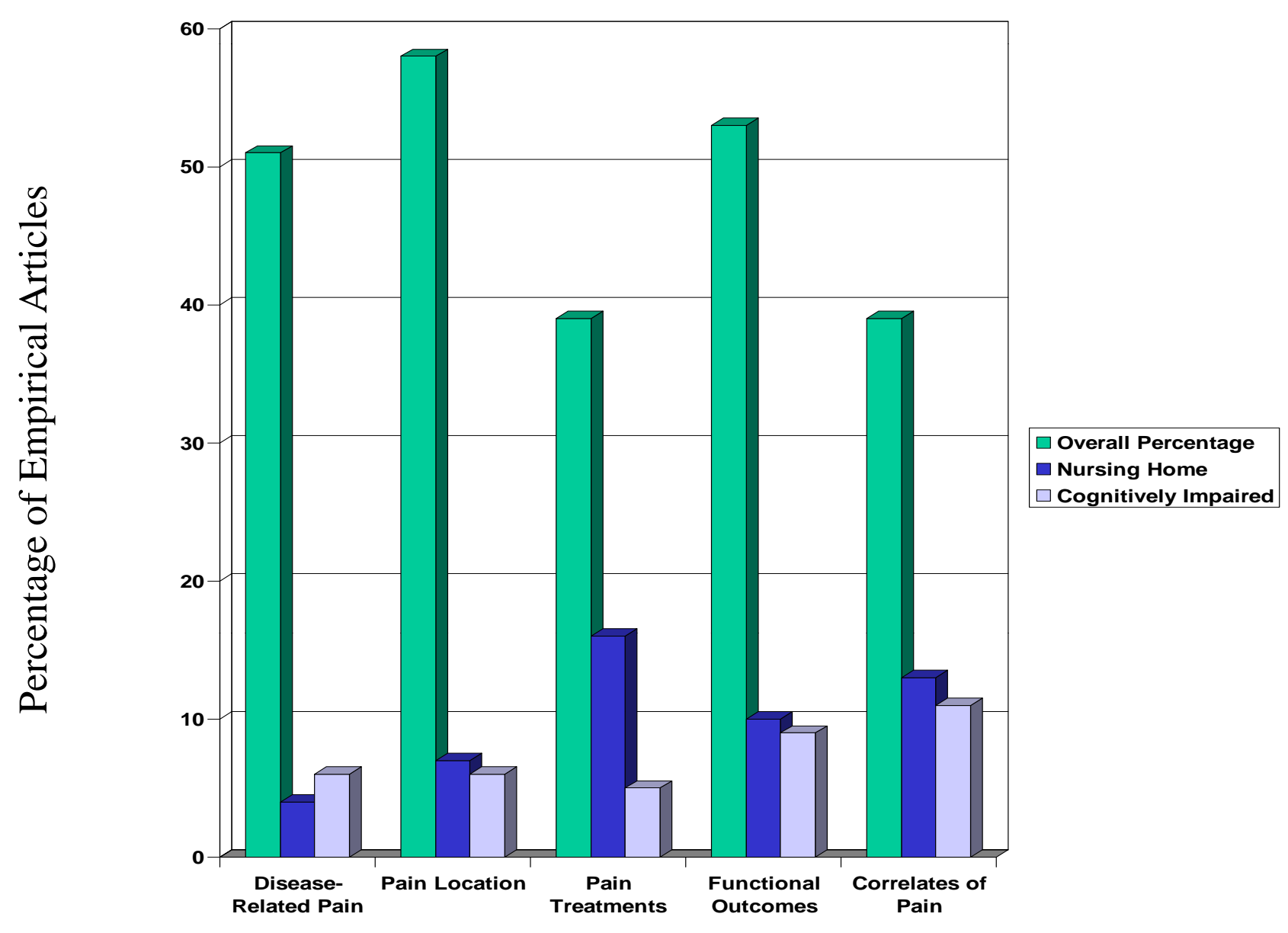

